\title{
Análisis de la inclusión educativa en contextos vulnerables
}

Educational Inclusion Analysis in Vulnerable Contexts

Análise da inclusão educativa em contextos vulneráveis

Andrés Escarbajal-Frutos* (iD htcps//orcid.org/0000-0001--7699-1240

Purificación Corbalán-Palazón** (iD hteps//orcid.org/0000-0002-4230-2539

Paloma Orteso-Iniesta *** (iD) hetps///orcidorg/0000-0003-3694-3150

Doi: 10.17227/rce.num78-6776

Para citar este artículo: Escarbajal, A., Corbalán, P., Orteso, P. (2O2O). Análisis de la inclusión educativa en contextos vulnerables. Revista Colombiana de Educación, 78, 361-382. http://doi.org/10.17227/rce.num78-6776

$\begin{array}{ll}\text { (C) } \bigcup_{\mathbf{B Y}} \$ \mathbf{N C} & \text { Recibido: 10/03/2017 } \\ \text { Evaluado: 17/09/2019 }\end{array}$

* Doctor Europeo en Pedagogía Social, Especialista en Mediación Intercultural, Profesor de la Facultad de Educación en la Universidad de Murcia, y asesor-consultor de prácticas y políticas educativas. Miembro del grupo de Investigación Educación Inclusiva: Escuela para Todos. Correo electrónico: andreses@um.es

** Máster en Educación Inclusiva por la Universidad de Murcia. Investigadora colaboradora en la Facultad de Educación de la Universidad de Murcia, especializada en contextos vulnerables en centros educativos. Correo electrónico: purificación.corbalán@um.es

*** Doctoranda en Educación por la Universidad de Murcia en el ámbito de Atención a la Diversidad y Educación Inclusiva, maestra especialista en Necesidades Específicas de Apoyo Educativo. Correo electrónico: palomaorteso@um.es 


\title{
Resumen
}

Partiendo de la necesidad de establecer un modelo educativo que responda a la sociedad dinámica en la que vivimos, como el modelo de la inclusión educativa, en este artículo derivado de una investigación más amplia, se expone el análisis de la capacidad de tres centros educativos de infantil y primaria, situados en la Región de Murcia (España), para desarrollar una enseñanza inclusiva. Para tal fin se llevó a cabo un estudio del contexto de dichos centros, a través de la implementación de una versión adaptada del cuestionario Autoevaluación de centros para la atención a la diversidad desde la inclusión (ACADI) desarrollado por Arnaiz y Guirao (2015). Los resultados muestran compatibilidad con el desarrollo de los ideales inclusivos en los centros estudiados; además, se registran diferencias estadísticamente significativas entre estos, que denotan la necesidad de seguir trabajando en la construcción de una sociedad inclusiva, especialmente en dos de ellos Del mismo modo, el estudio correlacional de valores y respuesta educativa pone de manifiesto correlaciones positivas entre mayores niveles de competencia inclusiva en los centros y el desarrollo de valores inclusivos en la comunidad educativa.

\section{Palabras clave}

inclusión; autoevaluación; atención a la diversidad calidad; proceso educativo

\section{Keywords}

inclusion; self-assessment; attention to diversity; quality; educational process

\begin{abstract}
Considering how important it is to stablish an educative system capable of responding to the dynamic society in which we life, as it is the educational inclusion approach, in this research article, which belongs to a larger investigation, is exposed the analysis of the capacity to develop an inclusive education, in three Elementary Schools in The Region of Murcia. For that aim, it was carried out an analysis of school's context, through an adapted version of the questionnaire "Self-evaluation of Centres for the Diversity Attention from Inclusion" which was developed by Arnaiz and Guirao (2015). The results show a compatibility with developing inclusive ideals in the studied centres, also have been detected statistically significant differences, which show the importance of continue working on the construction of an inclusive society. In the same way, the correlational study with regard to the educative community values and the educative response, reveals the existence of positive correlations between higher levels of inclusive competence in the schools and the development of inclusive values in the educational community.
\end{abstract}

\section{Resumo}

A partir da necessidade de estabelecer un modelo educativo que responda a sociedade dinâmica na qual vivemos, como o modelo da inclusão educativa, este artigo, derivado de pesquisa mais ampla, apresenta uma análise sobre a capacidade de três centros de educação infantil e básica situados na região da Múrcia (Espanha) para desenvolver uma educação inclusiva. Para tal, foi realizado um estudo sobre o contexto dos centros, através da implementação de uma versão adaptada do questionário Autoavaliação de centros para a atenção à diversidade a partir da inclusão (ACADI), desenvolvido por Arnaiz e Guirao (2015). Os resultados mostram compatibilidade com o desenvolvimento de ideais inclusivos nos centros pesquisados. Além disso, registram-se diferenças estatísticamente significativas entre eles, indicando a necessidade de continuar trabalhando na construção de uma sociedade inclusiva, especialmente em dois centros. Do mesmo modo, o estudo correlacional de valores e resposta educativa indica correlações positivas entre níveis mais altos e competência inclusiva nos centros e desenvolvimento de valores inclusivos na comunidade educativa.

\section{Palavras-chave}

inclusão; autoavaliação; atenção à diversidade; qualidade; processo educativo 


\section{Introducción}

La construcción de sociedades inclusivas donde imperen valores como el respeto, la democracia o la equidad (Arnaiz, De Haro y Maldonado, 2019), lleva consigo la necesidad por desarrollar escuelas capaces de educar en y para la diversidad, que sirvan como pilar de la consecución de sociedades más justas y equitativas (Pino y Castaño, 2019), donde las diferencias económicas, culturales o de cualquier índole no supongan una desventaja (Ossa, Castro, Castañeda y Castro, 2014).

Teniendo en cuenta esta idea, con el enfoque de la educación inclusiva se persigue el desarrollo de una enseñanza que garantice el éxito de todo el alumnado y permita su desarrollo pleno en la sociedad (Arnaiz, 2012). Siendo las siguientes concepciones la base de la inclusión:

» Que la educación sea entendida como un proceso que facilite el abordaje de la diversidad desde una visión positiva en la que las diferencias se consideran potencialidades para el sistema (Ainscow y Echeita, 2011; Arnaiz, 2011; Unesco, 2005).

» La lucha contra las barreras que impiden el desarrollo de la inclusión, así como la detección de estas, y las palancas para llegar al cambio de esas situaciones (Ainscow, 2004).

»Son principios fundamentales el aprendizaje, a través de aquellas medidas y actuaciones que garanticen su pleno desarrollo en el sistema educativo; la presencia y la participación de dichos alumnos $y$, en general, de toda la comunidad educativa teniendo en cuenta la diversidad y necesidades de todos (Echeita, 2006; Rodríguez, 2015; Ainscow, 2017).

En este sempiterno camino hacia la inclusión, la evaluación de la práctica educativa y del punto de partida de un contexto en concreto se hace indispensable (Escarbajal, Arnaiz y Giménez, 2017). Primero, porque no todas las comunidades educativas precisan de las mismas necesidades; y, segundo, porque aceptar que las sociedades son entes dinámicos implica la necesidad de una evaluación constante que facilite el planteamiento de objetivos y prácticas educativas ajustados a las necesidades del momento (Escarbajal, 2015).

De modo que estudiar el estado de una cuestión como la calidad inclusiva de un centro y su personal docente implica llevar a cabo una evaluación previa que brinde una imagen de las necesidades de dicho contexto y nunca partir de concepciones verosímiles (Guirao y Arnaiz, 2015).

Partiendo de esta premisa, ante la preocupación por el riesgo de exclusión social de grupos vulnerables existente en tres centros de la Región de Murcia, se aplicó la dimensión de contexto del cuestionario 
de Autoevaluación de centros para la atención a la diversidad desde la inclusión, desarrollado por Arnaiz y Guirao (2015), a fin de conocer la situación inclusiva de los centros, con un sistema de comprobada eficacia, y evaluar aquellos aspectos susceptibles de mejora.

\section{Los valores de una escuela inclusiva}

La educación inclusiva supone entender que una escuela que pretenda ser inclusiva deberá tener en cuenta a todo su alumnado; establecer su respuesta educativa según las necesidades de ese colectivo (Rodríguez, 2015; Escarbajal et al., 2012), y adoptar los tres principios inclusivos por excelencia: presencia, aprendizaje y participación (Ainscow, 2017; Echeita, 2006; Arnaiz, 2011; Padrós, Duque y Molina, 2011).

Sin embargo, para que dichos principios puedan ser una realidad en la escuela, es necesaria una concienciación que garantice la existencia de valores inclusivos en la comunidad (Pino y Castaño, 2019; Rodríguez, 2015).

En este sentido, de acuerdo con la investigación Improving the quality of education for all (IQEA), en la que participaron cerca de 40 escuelas de Reino Unido (Ainscow, Hopkins, Soutworth y West, 2001), se determinó que, para que las acciones emprendidas desde el centro se sostengan sobre una buena base inclusiva, deben asumirse los siguientes principios (tal y COmo se hizo en la experiencia IQEA):

» Tomar las necesidades de la comunidad educativa como referente para trazar el punto de partida, siendo las prioridades del centro las que su contexto determine.

» Involucrar a toda la comunidad educativa en la toma de decisiones y su aplicación. Para ello, establecer desde la institución educativa la creación de estructuras y mecanismos de coordinación que doten a los miembros de voz para participar.

» Favorecer en el profesorado la conciencia sobre la necesidad de evaluación de la calidad educativa como mecanismo para la garantía de una mejor enseñanza.

»Establecer las condiciones necesarias, desde la institución escolar, para que todo el alumnado alcance el aprendizaje. La consecución de dicha meta es responsabilidad de los centros y en ningún caso de las capacidades del alumnado o su entorno.

Sin embargo, cuando hablamos de valores o actitudes inclusivas, es pertinente resaltar otros aspectos actitudinales, aparte de los ya mencionados. Según Pérez-Cobacho y Prieto (2003), la escuela inclusiva deberá 
estar empapada de valores como "aceptación, sentimiento de pertenencia, comunidad, capacidades para los aprendizajes y relaciones personales [...] centrada en destacar las cualidades y las necesidades de cada uno [...] para que se sientan bienvenidos y seguros" (p. 72). En definitiva, hablamos de un enfoque teórico donde la concienciación y los valores propios de este son una parte muy significativa.

\section{Repuesta educativa. Generando entornos más inclusivos}

El trascurso de los años junto la evolución investigadora y la praxis profesional han dado lugar a una serie de cambios y avances en el panorama práctico-legislativo de la enseñanza que nos han Ilevado a pasar de una visión repudiante de la diversidad funcional o cultural (antigüedad clásica) a un modelo incluyente, social y educativo, donde todas las personas tienen los mismos derechos (Echeita, 2006).

Sin embargo, para que la inclusión sea una realidad, las escuelas deben constituirse en base a unos principios que aseguren una enseñanza acorde a la diversidad, basada en la calidad y equidad de su alumnado, que tome en cuenta a toda la comunidad educativa y ofrezca una respuesta que se ajuste a sus necesidades (Ainscow et al., 2001); como se expresó en el anterior apartado.

Desde los centros educativos, estos principios se ven reflejados a través de los tres niveles de concreción curricular (centro, aula e individual) que las escuelas establecen cada año para adecuar el currículum común estatal a las características del contexto en que se desempeña la enseñanza (Álvarez, 2011).

Por consiguiente, si tenemos en cuenta que la concreción curricular y su planificación de son la base para fomentar una escuela inclusiva, en la que todos sus alumnos alcancen el pleno desarrollo, cabría preguntarse: ¿Cuáles son aquellas prácticas que desde el centro y aula deben tenerse en cuenta, si deseamos educar desde un enfoque inclusivo?

En este sentido, los resultados de la investigación IQEA demostraron mayor éxito en aquellos centros donde se llevó a cabo la reflexión colectiva a la hora de determinar cambios; se estableció la comunicación coordinada entre profesionales trabajando de modo colaborativo, y donde existía una buena distribución del liderazgo directivo (Galeano, 2002).

Respecto a la organización escolar, es destacable el proyecto INCLU_ED (Flecha y Molina, 2013), en el que colaboraron 14 países europeos. En este se analizaron actuaciones que llevaban al éxito inclusivo. 
Entre las conclusiones de este proyecto, se demostró dentro del ámbito organizativo de centro y aula que la utilización de grupos heterogéneos y desdobles heterogéneos conlleva mayores niveles de inclusión. Así como la participación de familias en los grupos heterogéneos como apoyo hacia el aprendizaje, siendo un elemento enriquecedor (Flecha, García, Sordé y Redondo, 2006).

Atendiendo a la respuesta educativa y medidas para responder a la diversidad, son recomendadas las "adaptaciones curriculares individuales e inclusivas" (adecuar aspectos metodológicos para facilitar el aprendizaje) y la "optatividad inclusiva" (que se basa en los intereses y no en las capacidades). Estas parten siempre de que, en ningún momento, la responsabilidad recaiga en el alumno, sino que sea el centro quien se ocupe de responder a las necesidades (Rodríguez, 2015).

A modo de síntesis, ofrecer una respuesta educativa que favorezca la inclusión implica llevar a cabo mecanismos de coordinación y colaboración docente que ayuden a caminar en una misma dirección; así como la utilización de metodologías variadas que faciliten el acceso al aprendizaje, entre ellas: agrupamientos heterogéneos, adecuación de actividades o uso de diversos procedimientos para la evaluación (Escarbajal, Navarro y Arnaiz, 2019). Esto se logra sin olvidar la importancia de crear espacios físicos y administrativos accesibles y adecuados para toda la comunidad educativa, que permitan el desarrollo de su autonomía (Unesco, 2006).

\section{La autoevaluación como herramienta para mejorar}

En la línea de la evaluación sobre la inclusión educativa, a lo largo de los años han ido apareciendo indicadores encaminados a ofrecer una evaluación, formativa y procesual, que brinde a las instituciones un mecanismo de diagnóstico con el cual concretar futuras líneas de mejora y detectar aquellas situaciones de exclusión que han de ser abordadas (Kyriazopoulou y Weber, 2009).

Teniendo en cuenta la importancia de la evaluación en la planificación de la acción educativa, es importante retomar uno de los principios que señalábamos anteriormente: el desarrollo de la capacidad para evaluar la calidad educativa como eslabón para establecer un punto de partida y valoración del proceso (Ainscow, Hopkins, Soutworth y West, 2001).

De acuerdo con diversos autores (Arnaiz y Martínez, 2018; Mateo, 2000; Santos, 2004), la reflexión y evaluación de la práctica educativa desde el propio centro, servirán como herramienta para identificar los puntos fuertes y débiles de la institución escolar a la hora de garantizar la inclusión del alumnado. 
En este ámbito, se destacan los instrumentos de autoevaluación y evaluación de las diversas dimensiones inclusivas que permiten al profesorado tener una imagen de la situación de su centro, a fin de identificar las necesidades del entorno.

En este sentido, en el contexto de la Región de Murcia (España), existe el instrumento Autoevaluación de centros para la atención a la diversidad desde la inclusión (ACADI), de Guirao y Arnaiz (2015), cuya validez se sustenta en un trabajo de investigación del que formaron parte un gran número de expertos y que se fundamentó en otros instrumentos, como el index for inclusión (Booth y Ainscow, 2002).

Dicha herramienta de autoevaluación consiste en un cuestionario basado en cuatro ámbitos en los que se analizan el contexto escolar, recursos, resultados y proceso educativo. Con su planteamiento se formulan afirmaciones propias del enfoque inclusivo que los docentes, de modo individual, deben responder, y así, mostrar su nivel de coincidencia con la expresión propuesta mediante la escala Likert de 1 a 4 (1: muy poco; 2: poco; 3: bastante; 4: mucho).

Tras la aplicación de dicho cuestionario se dispone de un conjunto de indicadores (con consistencia interna) que posibilitan la evaluación de la calidad educativa a través de la reflexión y la autoevaluación; así, se detectan aquellas actuaciones de mejora para avanzar en la consecución de contextos más inclusivos (Arnaiz y Guirao, 2015).

\section{Objetivos de la investigación}

El objetivo del presente estudio pasa por analizar la relación existente entre el desarrollo de valores inclusivos en la comunidad educativa y, el tipo de respuesta educativa de centros de educación infantil y primaria ubicados en contextos vulnerables, a través de la autoevaluación docente. Para su concreción se proponen los siguientes objetivos específicos:

» Objetivo específico 1 (OE1): Conocer el estado de la cuestión relativa a valores inclusivos compartidos por la comunidad educativa.

» Objetivo específico 2 (OE2): Valorar el grado en que la respuesta educativa, que se lleva a cabo en el centro, coincide con los principios de una escuela inclusiva.

» Objetivo específico 3 (OE3): Comprobar en qué medida la acción docente en el aula se ajusta a los principios inclusivos.

» Objetivo específico 4 (OE4): Analizar el tipo de correlación entre la respuesta educativa y los niveles de concienciación inclusiva en la comunidad educativa. 


\section{Metodología}

\section{Diseño}

La investigación se enmarca en la dimensión epistemológica del paradigma humanístico interpretativo, centrado en el estudio de factores relacionados con acciones de la vida y el ámbito social, a fin de interpretar el estado de una cuestión (Sarrado, Cléries, Ferrer y Kronfly, 2004; Nieto y Rodríguez, 2009). En cuanto a la dimensión metodológica, se trata de un estudio ex post facto, dado que las variables han sido analizadas sin haber realizado una intervención previa (Hernández, Fernández y Baptista, 2010). Así, el diseño del presente análisis es de corte cuantitativo no experimental, ya que pretende dar a conocer la relación entre ciertas variables, y la secuencia es de corte transversal, pues la recolección de datos se establece en un solo momento en el tiempo. Por ello, es una investigación descriptiva (Hernández, Fernández y Baptista, 2010), para medir los niveles en los que se presentan una o varias variables dentro de una población.

\section{Instrumento}

Para responder a los objetivos planteados, se ha utilizado el cuestionario de Autoevaluación de centros para la atención a la diversidad desde la inclusión (ACADI) (Arnaiz y Guirao, 2015), basado en cuatro dimensiones de análisis: a) contexto escolar, evaluando indicadores como los valores inclusivos compartidos por la comunidad educativa, los agrupamientos de los alumnos, el plan de acogida; b) recursos, en la que se estudian los criterios para determinar qué recursos son necesarios, la finalidad en su dotación, instalaciones y materiales; c) proceso educativo, la existencia de metodologías adoptadas, el uso de refuerzos y apoyos para atender a la diversidad; y d) resultados, si obtienen las metas preestablecidas, si mejora progresivamente el alumnado, el clima de convivencia. Su planteamiento consiste en la formulación de ítems propios del enfoque inclusivo que los docentes, de modo individual, deben responder, mediante la escala Likert de 1 a 4 (1: muy poco; 2: poco; 3: bastante; 4: mucho). En concreto, se han empleado indicadores de la dimensión 1 (contexto) de dicho cuestionario que se compone de 14 categorías donde se abordan aspectos como: valores inclusivos, convivencia, coordinación y colaboración, etc. Dentro de dichas categorías se recogen los indicadores que permiten evaluar la situación del centro.

\section{Procedimiento y periodo de realización de la investigación}

En lo que refiere al periodo de realización de esta investigación, teniendo en cuenta la construcción del instrumento anteriormente comentado, y con la puesta en marcha de un estudio de los ítems contemplados en el 
ámbito contexto del cuestionario ACADI, con los que se seleccionaron los propuestos en el mismo, a fin de agilizar la cumplimentación por parte del profesorado, y explicados en el aparatado resultados, la duración fue de un año académico, pues el desplazamiento a los centros para depositar el cuestionario y otro desplazamiento para su recolección retrasaron los plazos establecidos.

\section{Análisis de datos}

Una vez seleccionados, se comprobó la fiabilidad de la elección a través de la prueba de fiabilidad alfa de Cronbach para constatar que la elección no implicaba alteración en los resultados, como puede verse en la tabla 1. De dicha prueba se obtuvieron resultados superiores a $\alpha=0,9$, lo cual denota altos niveles de fiabilidad en el cuestionario, en consecuencia, la elección de preguntas no supone una alteración.

Tabla 1.

Estadísticas alfa de Crombach de fiabilidad

\begin{tabular}{cc}
\hline Alfa de Cronbach & No. de elementos \\
\hline 0,932 & 17 \\
\hline
\end{tabular}

Fuente: elaboración propia.

En la aplicación del cuestionario, para la selección de la muestra se estableció como único criterio que fueran centros con alumnado en riesgo de exclusión o con colectivos vulnerables. A partir de dicho criterio, se estableció contacto con los directores de seis centros, a quienes se les propuso la participación en la investigación. Tras obtener respuesta positiva de tres centros, se les trasladó el cuestionario en formato impreso para su cumplimentación, de forma individual y sin contar con presión temporal que pudiera repercutir en los resultados.

Atendiendo al análisis de los datos, estos han sido procesados a través del software estadístico SPSS (IBM, 2013); para la obtención de resultados se aplicaron diversas pruebas estadísticas que permitieron la descripción de la información registrada, como son: el uso de frecuencias (destinado a conocer las características de la muestra), la media (a fin de identificar la tendencia de los grupos) y la desviación típica (conocer la dispersión de la media). Así mismo, también se han utilizado estadísticos de tipo inferencial, como la prueba ANOVA (comparar medias) y la correlación de Pearson (a la hora de estudiar la relación entre variables). 


\section{Participantes}

Han formado parte de la investigación un total de 78 docentes de educación infantil y primaria, que se distribuyen en tres centros de la Región de Murcia, caracterizados por una notoria diversidad cultural y funcional, o encontrarse en entornos en riesgo de exclusión social, como se describe en la tabla 2.

\section{Tabla 2.}

Características de los centros

\begin{tabular}{cl}
\hline Centro & \multicolumn{1}{c}{ Características relevantes para el estudio } \\
\hline & $\begin{array}{l}\text { Centro público de infantil y primaria de una sola línea, ubicado en un contexto } \\
\text { urbano, nivel socioeconómico medio, importante diversidad en el alumnado } \\
\text { con presencia significativa de alumnado con necesidades educativas especia- } \\
\text { les (NEE). Presencia de familias inmigrantes con bajos ingresos y desconoci- } \\
\text { miento del idioma. }\end{array}$ \\
\hline 3 & $\begin{array}{l}\text { Centro público, cercano al núcleo urbano que cuenta con más de una línea, } \\
\text { preferente de altas capacidades, población con un nivel socioeconómico entre } \\
\text { bajo y muy bajo. }\end{array}$ \\
\hline 3 & $\begin{array}{l}\text { Colegio público de infantil y primaria que cuenta con un aula abierta; dispone } \\
\text { de servicio de orientación una vez por semana toda la mañana completa. Es } \\
\text { notoria la presencia de alumnado con neE. }\end{array}$ \\
\hline
\end{tabular}

Fuente: elaboración propia.

Dentro de las características de la muestra (tabla 1), se destaca una proporción de docentes por centros similar, entre 26-30 docentes por colegio, siendo levemente superior en el centro 3 , mientras que la proporción entre hombres y mujeres es desigual, con superioridad de participación en las segundas.

\section{Tabla 3.}

Participantes de la investigación

\begin{tabular}{|c|c|c|c|c|c|c|c|c|c|}
\hline & \multicolumn{3}{|c|}{ Centro 1} & \multicolumn{3}{|c|}{ Centro 2} & \multicolumn{3}{|c|}{ Centro 3} \\
\hline & ixim & pipin & & imì & pipip & & iोi & piph & 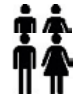 \\
\hline No. & 16 & 10 & 26 & 16 & 6 & 22 & 21 & 9 & 30 \\
\hline$\%$ & 20,5 & 12,8 & 33,3 & 20,5 & $7,7 \%$ & 28,2 & 26,92 & 11,54 & 38,46 \\
\hline
\end{tabular}

Fuente: elaboración propia. 


\section{Resultados}

Para la expresión de los resultados recurriremos a los objetivos específicos ya expuestos.

\section{Objetivo específico 1 (OEl): Conocer el estado de la cuestión relativa a valores inclusivos compartidos por la comunidad educativa}

A la hora de explorar los valores inclusivos en la comunidad educativa se utilizaron los ítems que aparecen en la tabla 4, los cuales guardan relación con aspectos como convivencia, respeto y concienciación con la diversidad.

Tabla 4.

Indicadores planteados para el ámbito estudiado

\begin{tabular}{|c|c|c|}
\hline $\begin{array}{c}\text { Aspecto } \\
\text { analizado }\end{array}$ & Afirmaciones propuestas & $\begin{array}{c}\text { Opciones de } \\
\text { respuesta }\end{array}$ \\
\hline $\begin{array}{l}\text { Valores } \\
\text { comunidad } \\
\text { educativa }\end{array}$ & $\begin{array}{l}\text { El profesorado tiene una actitud favorable a la incor- } \\
\text { poración de alumnado con necesidades especiales o } \\
\text { características diferentes al centro. } \\
\text { El alumnado intenta ayudar a los compañeros que tienen } \\
\text { más dificultades. } \\
\text { Existe un buen clima de convivencia en el centro entre } \\
\text { alumnos, profesionales y padres. } \\
\text { El profesorado intenta eliminar todas las barreras al } \\
\text { aprendizaje y la participación en el centro. } \\
\text { El centro se esfuerza por disminuir las prácticas discrimi- } \\
\text { natorias. } \\
\text { Se valoran las diferencias entre el alumnado, en vez de } \\
\text { tender a considerarlo como un grupo homogéneo. } \\
\text { El profesorado y otros profesionales, tanto antiguos como } \\
\text { de nuevo ingreso, se sienten satisfechos de la acogida al } \\
\text { comienzo en el centro escolar. }\end{array}$ & $\begin{array}{l}\text { 1: muy poco } \\
\text { 2: poco } \\
\text { 3: bastante } \\
\text { 4: mucho }\end{array}$ \\
\hline
\end{tabular}

Fuente: elaboración propia.

El análisis descriptivo de las respuestas que el profesorado aportó a nivel general (todos los centros), recogido en la tabla 5, muestra unos valores medios situados en 3,17 , con una desviación de casi medio punto que pone de manifiesto diferencias entre los centros, según se observa en los resultados por centro. 
Tabla 5.

Estadísticos descriptivos del oEl

\begin{tabular}{cccccccc}
\hline Centro & No. & Media & Desviación & $\begin{array}{c}\text { Límite } \\
\text { inferior }\end{array}$ & $\begin{array}{c}\text { Limite } \\
\text { superior }\end{array}$ & Mínimo & Máximo \\
\hline 1 & 26 & 3,6044 & 0,24347 & 3,5061 & 3,7027 & 2,86 & 4,00 \\
\cline { 2 - 7 } 2 & 22 & 2,7273 & 0,39410 & 2,5525 & 2,9020 & 2,29 & 3,57 \\
\cline { 2 - 7 } 3 & 30 & 3,1190 & 0,32291 & 2,9985 & 3,2396 & 2,57 & 3,71 \\
\cline { 2 - 7 } Todos & 78 & 3,1703 & 0,47075 & 3,0642 & 3,2765 & 2,29 & 4,00 \\
\hline
\end{tabular}

Fuente: elaboración propia.

En este sentido, los resultados del centro 1 son notoriamente superiores a los del resto de centros, con puntuación por encima de 3,5, por lo que los valores inclusivos en este centro son muy buenos. Las puntuaciones más bajas se encuentran en el centro 2, debajo del valor 3, lo cual denota una necesidad por mejorar la situación. Respecto al centro 3, las puntuaciones oscilan el 3,1, lo cual sería considerado mejorable.

Para comprobar si estas diferencias son estadísticamente significativas, se llevó a cabo la prueba ANOvA que permite comparar una misma variable en muchos grupos (Sáez, 2017), comprobando previamente que existía homogeneidad entre las medias a través de la prueba Brown- Forsythe, de la que se obtuvo el valor sig. 0,00 que indica que los grupos son homogéneos (tabla 6).

Tabla 6.

Comparación de medias ANOVA

\begin{tabular}{lccccc}
\hline & Suma de cuadrados & gl & Media cuadrática & F & Sig. \\
\hline Entre grupos & 9,296 & 2 & 4,648 & 44,881 & 0,000 \\
\cline { 2 - 5 } Dentro de grupos & 7,767 & 75 & 0,104 & \\
Total & 17,064 & 77 & & \\
\hline
\end{tabular}

Fuente: elaboración propia.

Como se observa, se confirma la existencia de diferencias estadísticas significativas que denotarían que los valores inclusivos entre un centro y otro no están igualmente arraigados. Sin embargo, esta prueba no permite saber entre qué grupos se hayan las diferencias; por este motivo, se llevó a cabo la prueba post hoc Bonferroni (tabla 7) que permite hacer comparaciones dos a dos. 
Tabla 7.

Prueba Bonferroni comparaciones múltiples

\begin{tabular}{cccccccc}
\hline \multirow{2}{*}{$\begin{array}{l}\text { (I) Centro } \\
\text { educativo }\end{array}$} & $\begin{array}{c}\text { (J) Centro } \\
\text { educativo }\end{array}$ & $\begin{array}{c}\text { Diferencia de } \\
\text { medias (I-J) }\end{array}$ & $\begin{array}{c}\text { Error } \\
\text { estándar }\end{array}$ & Sig. & \multicolumn{2}{c}{$\begin{array}{c}\text { 95\% de intervalo } \\
\text { de confianza }\end{array}$} \\
\cline { 6 - 8 } & & & & $\begin{array}{c}\text { Límite } \\
\text { inferior }\end{array}$ & $\begin{array}{c}\text { Límite } \\
\text { superior }\end{array}$ \\
\hline Centro 1 & Centro 2 & $0,87712^{*}$ & 0,09322 & 0,000 & 0,6488 & 1,1054 \\
\hline Centro 1 & Centro 3 & $0,48535^{*}$ & 0,08623 & 0,000 & 0,2742 & 0,6965 \\
\hline Centro 2 & Centro 3 & $-0,39177^{*}$ & 0,09033 & 0,000 & $-0,6130$ & $-0,1706$ \\
\hline
\end{tabular}

Fuente: elaboración propia.

Se desprende, por tanto, que las diferencias en los valores medios obtenidos en los tres centros son estadísticamente significativas, y por consiguiente los valores inclusivos de la comunidad educativa del centro 1 están más presentes que los del 2 y 3.

\section{Objetivo específico 2 (OE2): Valorar el grado en que la respuesta educativa que a nivel de centro se lleva a cabo coincide con los principios de una escuela inclusiva}

El desarrollo de este objetivo se llevó a cabo mediante los ítems expuestos en la tabla 8. Como se observa, todos ellos versan sobre aspectos relacionados con medidas, actuaciones educativas o visión de la diversidad en el centro.

\section{Tabla 8.}

Indicadores planteados para el ámbito estudiado

\begin{tabular}{|c|c|c|}
\hline $\begin{array}{l}\text { Aspecto } \\
\text { analizado }\end{array}$ & Afirmaciones propuestas & $\begin{array}{l}\text { Opciones } \\
\text { respuesta }\end{array}$ \\
\hline \multirow{7}{*}{$\begin{array}{l}\text { Respuesta } \\
\text { educativa y } \\
\text { principios del } \\
\text { centro }\end{array}$} & \multirow{4}{*}{$\begin{array}{l}\text { Se prefieren las formas de apoyo ordinarias (para } \\
\text { todos) frente a las específicas, utilizando estas solo } \\
\text { cuando las primeras no han funcionado. } \\
\text { El criterio básico para construir los grupos-clase es } \\
\text { la heterogeneidad (de género, de capacidades, de } \\
\text { intereses...). }\end{array}$} & 1: muy poco \\
\hline & & 2: poco \\
\hline & & 3: bastante \\
\hline & & 4: mucho \\
\hline & $\begin{array}{l}\text { Se considera la accesibilidad como base para incluir a } \\
\text { todas las personas con discapacidad de la comunidad } \\
\text { educativa. }\end{array}$ & \\
\hline & $\begin{array}{l}\text { La dotación de zonas de recreo y juego están debida- } \\
\text { mente acondicionadas y protegidas para la prevención } \\
\text { de accidentes. }\end{array}$ & \\
\hline & $\begin{array}{l}\text { Se adaptan a los horarios en función de las característi- } \\
\text { cas y necesidades del grupo. }\end{array}$ & \\
\hline
\end{tabular}


Los resultados para estos ítems, por centro y en su totalidad, han sido registrados en la tabla 9, donde se ha recogido el análisis descriptivo de los datos. Allí se observan unos valores medios generales ubicados cerca del valor 3, lo cual quiere decir que en el cómputo total prima el valor bastante de acuerdo, con el modo de establecer la respuesta educativa en lo que a su organización (indicadores: 9, 11 y 12) y su gestión se refiere (indicadores: 8 y 10). Se responde inclusivamente a la diversidad, en los aspectos organizativos y espaciales, aunque estos resultados son mejorables.

Tabla 9.

Estadísticos descriptivos del OE2

\begin{tabular}{cccccccc}
\hline Centro & No. & Media & Desviación & $\begin{array}{c}\text { Límite } \\
\text { inferior }\end{array}$ & $\begin{array}{c}\text { Límite } \\
\text { superior }\end{array}$ & Mínimo & Máximo \\
\hline 1 & 26 & 3,4846 & 0,40069 & 3,3228 & 3,6465 & 2,40 & 4,00 \\
2 & 22 & 2,6273 & 0,47528 & 2,4165 & 2,8380 & 1,60 & 3,60 \\
3 & 30 & 2,9333 & 0,46486 & 2,7598 & 3,1069 & 2,00 & 3,80 \\
todos & 78 & 3,0308 & 0,56115 & 2,9043 & 3,1573 & 1,60 & 4,00 \\
\hline
\end{tabular}

Fuente: elaboración propia.

Al analizar de modo pormenorizado la respuesta educativa de cada centro, se observan unos valores superiores en el centro 1 sobre el resto de los centros (una puntuación media de 3,48), siendo el centro 2 el de peores puntuaciones, cuyos valores medios no alcanzan la puntuación 3 (bastante).

Cuando se aplica la prueba ANOva (tabla 10) para la comprobación de diferencias entre grupos, de nuevo se hallan diferencias estadísticas significativas, que confirman que la respuesta educativa del centro entre un colegio y otro no coincide por igual con el enfoque inclusivo.

Tabla 10.

Comparación de medias ANOVA

\begin{tabular}{lccccc}
\hline & $\begin{array}{c}\text { Suma de } \\
\text { cuadrados }\end{array}$ & gl & Media cuadrática & F & Sig. \\
\hline Entre grupos & 9,222 & 2 & 4,611 & 23,018 & 0,000 \\
\cline { 2 - 6 } $\begin{array}{l}\text { Dentro de grupos } \\
\text { Total }\end{array}$ & 15,024 & 75 & 0,200 & & \\
\cline { 2 - 6 } & 24,246 & 77 & & \\
\hline
\end{tabular}

Fuente: elaboración propia.

Como se observa, se confirma que estas diferencias estadísticamente significativas, con un nivel de significación de 0,000. Para comprobar si dichas diferencias también son entre centros, se aplicó la prueba Bonferroni (tabla 11). 
Tabla 11.

Prueba Bonferroni comparaciones múltiples

\begin{tabular}{cccccccc}
\hline \multirow{2}{*}{$\begin{array}{c}\text { (I) Centro } \\
\text { educativo }\end{array}$} & $\begin{array}{c}\text { (J) Centro } \\
\text { educativo }\end{array}$ & $\begin{array}{c}\text { Diferencia de } \\
\text { medias (I-J) }\end{array}$ & $\begin{array}{c}\text { Error } \\
\text { estándar }\end{array}$ & Sig. & \multicolumn{2}{c}{$\begin{array}{c}\text { 95\% de intervalo } \\
\text { de confianza }\end{array}$} \\
\hline \multirow{2}{*}{$\begin{array}{c}\text { Centro 1 } \\
\text { Centro 1 }\end{array}$} & Centro 2 & $0,85734^{*}$ & 0,12965 & 0,000 & 0,5398 & $\begin{array}{c}\text { Límite } \\
\text { inferior }\end{array}$ & $\begin{array}{c}\text { Límite } \\
\text { superior }\end{array}$ \\
\cline { 2 - 7 } & Centro 3 & $0,55128^{*}$ & 0,11993 & 0,000 & 0,2576 & 0,8450 \\
\hline Centro 2 & Centro 3 & $-0,30606$ & 0,12563 & 0,052 & $-0,6137$ & 0,0016 \\
\hline
\end{tabular}

Fuente: elaboración propia.

De dicha tabla se desprende que existen diferencias estadísticamente significativas entre los centros 1 y 2, y los centros 1 y 3; por tanto, los resultados del centro 1 son superiores a los del resto.

Las diferencias entre los centros 2 y 3 se encuentran en el límite de significación $(0,052)$, por lo que la respuesta educativa del centro 3 parece más afín a un enfoque inclusivo que la del centro 2 .

\section{Objetivo específico 3 (OE3): Comprobar en qué medida la acción docente a nivel de aula se ajusta a los principios inclusivos.}

Para el estudio del tercer objetivo se plantearon las afirmaciones que se detallan en la tabla 12, relacionadas con adecuación curricular, respuesta metodológica y criterios de adaptación en el aula e individual.

\section{Tabla 12.}

Indicadores planteados para el ámbito estudiado

\begin{tabular}{|c|c|c|}
\hline $\begin{array}{c}\text { Aspecto } \\
\text { analizado }\end{array}$ & Afirmaciones propuestas & $\begin{array}{l}\text { Opciones } \\
\text { respuesta }\end{array}$ \\
\hline $\begin{array}{l}\text { Adecuación } \\
\text { curricular }\end{array}$ & $\begin{array}{l}\text { Se establecen criterios claros y consensuados por el } \\
\text { equipo de etapa para la elaboración de las programa- } \\
\text { ciones de aula. } \\
\text { Se adecuan las actividades programadas para dar } \\
\text { respuesta a las necesidades educativas especiales de } \\
\text { los alumnos en el proceso de enseñanza/aprendizaje. } \\
\text { Se utilizan diversos procedimientos e instrumentos } \\
\text { para evaluar el aprendizaje de los alumnos. } \\
\text { Se llevan a cabo distintos tipos de agrupamientos de } \\
\text { alumnos para favorecer el proceso de enseñanza/ } \\
\text { aprendizaje. } \\
\text { Se aplican actividades destinadas a promover el desa- } \\
\text { rrollo y autonomía de los alumnos en el centro. }\end{array}$ & $\begin{array}{l}\text { 1: muy poco } \\
\text { 2: poco } \\
\text { 3: bastante } \\
\text { 4: mucho }\end{array}$ \\
\hline
\end{tabular}


Según se muestra en la tabla 13, sobre análisis descriptivo, en el plano general (fila "todos") parece existir una tendencia a aplicar medidas que favorecerían la inclusión del alumnado, dado que los valores se sitúan en bastante $(3,14)$; sin embargo, la existencia de una desviación de 0,56 y la diferencia de dos puntos entre mínimo y máximo, hace pensar que existen diferencias entre los centros y esta respuesta no es igual de inclusiva en todos.

Tabla 13.

Estadísticos descriptivos del OE2

\begin{tabular}{cccccccc}
\hline Centro & No. & Media & Desviación & $\begin{array}{c}\text { Límite } \\
\text { inferior }\end{array}$ & $\begin{array}{c}\text { Límite } \\
\text { superior }\end{array}$ & Mínimo & Máximo \\
\hline 1 & 26 & 3,6923 & 0,28972 & 3,5753 & 3,8093 & 3,00 & 4,00 \\
\hline 2 & 22 & 2,5818 & 0,39957 & 2,4047 & 2,7590 & 2,00 & 3,60 \\
\hline 3 & 30 & 3,0867 & 0,38839 & 2,9416 & 3,2317 & 2,40 & 4,00 \\
\hline Todos & 78 & 3,1462 & 0,56630 & 3,0185 & 3,2738 & 2,00 & 4,00 \\
\hline
\end{tabular}

Fuente: elaboración propia.

En este sentido, cuando se estudian en detalle estos resultados a nivel de centro, vuelven a encontrarse valores similares a los que se obtuvieron en el primer objetivo; donde el centro 1 es superior $(3,69)$ sobre el $2(2,58)$ y el $3(3,08)$, tanto en las puntuaciones medias como en la dispersión de media, hecho que denota mayor similitud de respuestas entre los docentes del centro $1(0,28)$ que los del $2(0,39)$ y $3(0,38)$.

Del mismo modo que se hizo con los anteriores objetivos, para comprobar la importancia de estas diferencias se aplicó la prueba ANOVA, acompañada de la prueba la prueba post hoc Bonferroni para comparar entre grupos (tablas 14 y 15).

Tabla 14.

Comparación de medias ANOVA

\begin{tabular}{cccccc}
\hline & Suma de cuadrados & gl & Media cuadrática & F & Sig. \\
\hline Entre grupos & 14,868 & 2 & 7,434 & 56,743 & 0,000 \\
\cline { 2 - 5 } $\begin{array}{c}\text { Dentro de grupos } \\
\text { Total }\end{array}$ & 9,826 & 75 & 0,131 & \\
\cline { 2 - 5 } & 24,694 & 77 & & \\
\end{tabular}

Fuente: elaboración propia. 
Como vemos, las diferencias encontradas entre las medias obtenidas por los centros son estadísticamente significativas con un nivel de significación de 0,000 entre todos los centros, por lo que la respuesta en aula es significativamente más inclusiva en un centro que en otro. En la tabla 15 se esbozan dichas diferencias.

Tabla 15.

Prueba Bonferroni comparaciones múltiples

\begin{tabular}{ccccccc}
\hline $\begin{array}{l}\text { (I) Centro } \\
\text { educativo }\end{array}$ & $\begin{array}{c}\text { (J) Centro } \\
\text { educativo }\end{array}$ & $\begin{array}{c}\text { Diferencia de } \\
\text { medias (I-J) }\end{array}$ & $\begin{array}{c}\text { Error } \\
\text { estándar }\end{array}$ & Sig. & $\begin{array}{c}\text { 95\% de intervalo de confianza } \\
\text { Limite inferior }\end{array}$ & Límite superior \\
\hline Centro 1 & Centro 2 & $1,11049^{*}$ & 0,10485 & 0,000 & 0,8537 & 1,3673 \\
Centro 1 & Centro 3 & $0,60564^{*}$ & 0,09698 & 0,000 & 0,3681 & 0,8431 \\
Centro 2 & Centro 3 & $-0,50485^{*}$ & 0,10160 & 0,000 & $-0,7536$ & $-0,2560$ \\
\hline
\end{tabular}

Fuente: elaboración propia.

Observando los resultados expuestos en la columna "Sig." de la tabla 14 podemos deducir que la respuesta educativa en el centro 1 se ajusta en mayor medida al modelo inclusivo que la ofrecida por los centros 2 y 3 , de modo que se deberían aplicar mejoras en la respuesta educativa de dichos centros que los hagan caminar a puntuaciones similares al centro 1 .

\section{Objetivo específico 4 (OE4): Analizar el tipo de correlación existente entre la respuesta educativa y los niveles de concienciación inclusiva en la comunidad educativa}

El estudio correlacional permite explicar en qué medida una variable explicaría otras (Toro y Parra, 2006). En este caso, para conocer el tipo de correlación entre los valores de la comunidad educativa y la calidad de la respuesta educativa, se llevó a cabo la prueba de relación lineal entre variables correlación de Pearson (tabla 16).

Tabla 16.

Correlación de Pearson

\begin{tabular}{cccc}
\hline & & Respuesta nivel centro & Adecuación currículo \\
\hline \multirow{2}{*}{ Valores } & Correlación de Pearson & $0,700^{* *}$ & $0,760^{* *}$ \\
\cline { 2 - 4 } & Sig. (bilateral) & 0,000 & 0,000 \\
\cline { 2 - 4 } & No. & 78 & 78 \\
\hline
\end{tabular}

** La correlación es significativa en el nivel 0,0l (2 colas).

Fuente: elaboración propia. 
Los resultados muestran una correlación positiva y significativa entre las variables, con valores que oscilan entre el $70 \%-75 \%$ en todas las dimensiones, de manera que se confirmaría que la existencia de valores inclusivos en un centro implica una mejor respuesta educativa (desde el punto de vista inclusivo), y viceversa.

\section{Discusión y conclusiones}

La aplicación de la autoevaluación relativa al estudio de valores y respuesta educativa, a través del cuestionario ACADI, permite conocer la realidad de centros de enseñanza y disponer de unos resultados objetivos que posibilitan continuar el camino hacia la construcción de escuelas inclusivas (Arnaiz y Guirao, 2015).

En la presente investigación, ha sido posible evaluar la respuesta a la diversidad y los valores inclusivos existentes en tres centros de la Región de Murcia, atendiendo a una parte de la dimensión contexto educativo, recogida en el cuestionario ACADI.

Dentro de las conclusiones extraídas, a nivel general, coincidientes con la investigación de Escarbajal, Arnaiz y Giménez (2017), parece existir en el colectivo docente una preocupación por la inclusión del alumnado, es decir, los docentes son conscientes de la necesidad de que todos los alumnos alcancen el pleno desarrollo.

Del mismo modo, se percibe cierta concienciación sobre el respeto y tolerancia entre el alumnado, aunque los análisis de las prácticas educativas ponen de manifiesto la necesidad de seguir trabajando en la construcción de entornos más inclusivos, sobre todo en los centros 2 y 3.

Respecto a las aplicaciones educativas de este trabajo, que como se mencionó forman parte de un estudio más amplio donde también se analizaron aspectos como el liderazgo pedagógico o las aptitudes docentes, se pueden extraer las siguientes conclusiones:

» Invertir en políticas de concienciación sobre la diversidad y la importancia de la educación en ella ayudará a generar profesionales con mayores niveles de implicación a la hora de desempeñar su labor.

» La aplicación del instrumento ACADI, con un adecuado sistema de análisis de los resultados, ofrece una visión objetiva de la situación inclusiva de un entorno educativo.

» A la hora de definir los objetivos del proyecto educativo de un centro, así como las vías de actuación para alcanzarlos, los resultados ofrecidos por ACADI permiten detectar los aspectos en los que se debe incidir, como en el caso de esta investigación se observó 
que los centros 2 y 3 debían plantearse nuevas estrategias para la respuesta educativa en todos los niveles.

Profundizando en el análisis comparativo intercentros, conforme lo estudiado en los OE1, OE2 y OE3, los resultados indican la existencia de diferencias estadísticas significativas, tanto en la respuesta educativa (centro y aula) como en los valores de los tres centros estudiados. Este dato puede ser de mucha utilidad a la hora de establecer un plan de mejora tendente a la inclusión del alumnado.

Concretamente, se ha detectado una necesidad de mejora en los centros 2 y 3, cuyas puntuaciones en todos los ámbitos son significativamente inferiores al centro 1, y se hallan por debajo de 3 o en el límite. Si sometemos estos resultados a las categorías atribuidas en la investigación realizada por Escarbajal, Arnaiz y Giménez (2017), en la que los valores inferiores a tres son considerados mejorables (debilidades); 3 y 3,4, aceptables, y 3,4 en adelante, buenos; se puede afirmar que los centros 2 y 3 deberían emprender acciones que les hagan potencialmente inclusivos, sobre todo en el ámbito de la praxis educativa.

Como aspecto positivo del estudio comparativo, a la hora de confeccionar una hoja de ruta hacia la construcción de entornos más inclusivos en los centros 2 y 3 , podría ser un referente el proyecto educativo y las actuaciones que se emprenden en el centro 1.

Se destaca en esta línea que, siendo un centro más pequeño y con una importante diversidad funcional y cultural, sus resultados son más alentadores. Lo cual podría invitar a reflexionar sobre las limitaciones de los macrocentros, puesto que ha sido el centro con menos recursos y menos profesionales el que mejores puntuaciones ha logrado. Por esta razón, convendría estudiar en futuros trabajos la importancia de la masificación estudiantil a la hora de desarrollar escuelas inclusivas.

Respecto al estudio de las relaciones entre la respuesta pedagógica y los valores inclusivos en la comunidad educativa, desarrollado en el objetivo OE4, se observa que, a mayores puntuaciones dentro de la categoría valores inclusivos en docentes, discentes y familias, la respuesta educativa resulta más afín a la de la filosofía inclusiva. Es decir, un centro donde exista un profesorado concienciado con la diversidad y con un buen clima de convivencia tiene más posibilidades de ofrecer una respuesta que facilite el éxito de todos los alumnos, que aquel donde no exista cierta conciencia.

Dicha conclusión coincide con el estudio Learning without limits (Hart, Dixon, Drummond y McIntyre, 2004), donde se demostró que la convicción del profesorado influye en los avances de sus alumnos; y la investigación Understanding and developing inclusive practices (Ainscow, Booth y Dyson, 2006), en la que se indagó que la preocupación por la inclusión determina el tipo de respuesta que se ofrece al alumnado. 
Este hecho lleva a reflexionar sobre la importancia de una formación y concienciación en la diversidad en todos los niveles de la enseñanza, ya que, como demuestra el presente estudio, los valores de la comunidad educativa influyen en la respuesta inclusiva en un nivel de correlación del $75 \%$. Según este dato, una de las vías, si se quiere seguir desarrollando entornos inclusivos, debe ser la apuesta por actuaciones y planes de intervención destinados a generar conciencia sobre la diversidad en el profesorado y la comunidad educativa, lo cual repercutirá en la respuesta educativa y, en consecuencia, en el éxito escolar del alumnado.

\section{Referencias}

Ainscow, M. (2004). El desarrollo de sistemas educativos inclusivos: ¿Cuáles son las palancas de cambio? Journal of Educational Change. https://es.scribd.com/document/242980220/El-desarrollo-de-sistemas-educativos-inclusivos-pdf

Ainscow, M. (2017). Haciendo que las escuelas sean inclusivas: lecciones a partir del análisis de la investigación internacional. Revista de Educación Inclusiva, 5(1), 39-49.

Ainscow, M. y Echeita, G. (2011). La educación inclusiva como derecho. Marco de referencia y pautas de acción para el desarrollo de una revolución pendiente. Revista de Didáctica de la Lengua y la Literatura, 12(1), 26-46.

Ainscow, M., Booth, T. y Dyson, A. (2006). Improving schools, developing inclusion. Londres: Routledge.

Ainscow, M., Hopkins, D., Soutwoth, G. y West. M. (2001). Hacia escuelas eficaces para todos, manual para la formación de equipos docentes. Madrid: Narcea.

Álvarez, N. (2011). Niveles de concreción curricular. Revista Pedagogía Magna, 10(1), 151-158.

Arnaiz, P. (2011). Luchando contra la exclusión: buenas prácticas y éxito escolar. Revista de Innovación Educativa, 21, 23-35.

Arnaiz, P. (2012). Escuelas eficaces e inclusivas: cómo favorecer su desarrollo. Educatio Siglo XXI, 30(1), 25-44.

Arnáiz, P. y Guirao, J.M. (2015). La autoevaluación de centro en España para la atención a la diversidad desde una perspectiva inclusiva: ACADI. Revista Electrónica Interuniversitaria de Formación del Profesorado, 18(1), 45-101.

Arnaiz, P. y Martínez, P. (2018). Centros educativos que se autoevalúan y reflexionan sobre sus resultados para mejorar la atención a la diversidad. Revista Española de Orientación y Psicopedagogía, 29(1), 74-90. 
Arnaiz, P., De Haro, R. y Maldonado, R.M. (2019). Barriers to student learning and participation in an inclusive school as perceived by future education professionals. NAER: Journal of New Approaches in Educational Research, 8(1), 18-24.

Booth, T. y Ainscow, M. (2002). Index for inclusion. Bristol: Centre for Studies on Inclusive Education.

Echeita, G. (2006). Educación para la inclusión o educación sin exclusiones. En G. Echeita (ed.), ¿Por qué hablamos de educación inclusiva? La inclusión educativa como prevención de la exclusión social (pp. 75111). Madrid: Narcea.

Escarbajal, A. (2015). El derecho a la ciudadanía intercultural. En A. Escarbajal (ed.), Comunidades Interculturales y democráticas (pp. 39-52). Madrid: Narcea.

Escarbajal, A., Arnaiz, P. y Giménez, A. (2017). Evaluación de las fortalezas y debilidades del proceso educativo en centros de infantil, primaria y secundaria desde una perspectiva inclusiva. Revista Complutense de Educación, 28(2), 427-443.

Escarbajal, A., Mirete, A.B., Maquilón, J.J., Izquierdo, T., López, J.I., Orcajada, N. y Sánchez, M. (2012). La atención a la diversidad: la educación inclusiva. Revista Electrónica Interuniversitaria de Formación del Profesorado, 15(1), 135-144.

Escarbajal, A., Navarro, J. y Arnaiz, P. (2019). El rendimiento académico del alumnado autóctono y de origen inmigrante en la Región de Murcia. Tendencias Pedagógicas, 33(1), 5-17.

Flecha, J.R. y Molina, S. (2013). Aportaciones del proyecto INCLUD_ED a la mejora de la gestión educativa. Revista de Organización y Gestión Educativa, 5, 26-27.

Flecha, J.R., García, C., Sordé, T. y Redondo, G. (2006). Estrategias para la inclusión y la cohesión social en Europa desde la educación. En J. Giró (coord.), La escuela del siglo XXI: la educación en un tiempo de cambio social acelerado. XII Conferencia de sociología de la educación (pp. 129-130). Logroño, España.

Galeano, E. (2002). Modulo 8: Escuelas Eficaces con actitud. En R. Luna y J.A. Espinosa (Eeds.) Educación Inclusiva. Iguales en la diversidad. Recuperado de http://www.ite.educacion.es/formacion/materiales/126/ cd/unidad_8/mo8_introduccion.htm

Guirao, J.M. y Arnaiz, P. (2015). La educación intercultural en una escuela inclusiva. Vías para reorientar el camino. En A. Escarbajal (ed.), Comunidades interculturales y democráticas (pp. 39-52). Madrid: Narcea.

Hart, S., Dixon, A., Drummond, M.J. y Mclntyre, D. (2004). Learning without limits. Nueva York: McGraw-Hill

Hernández, R., Fernández, C. y Baptista, P. (2010). Metodología de la investigación. México D.F.: McGraw-Hill 
IBM (2013). Statistical Package for the Social Sciences (versión 22). [Software]. Nueva York.

Kyriazopoulou, M. y Weber, H. (2009). Desarrollo de indicadores sobre educación inclusiva en Europa. Odense: European Agency for Development in Special Needs Education.

Mateo, J. (2000). La evaluación educativa, su práctica y otras metáforas. Barcelona: ICEHorsori.

Nieto, S. y Rodríguez, M.J. (2009). Investigación y evaluación educativa en la sociedad del conocimiento. Salamanca: Ediciones Universidad de Salamanca.

Ossa, C., Castro, F., Castañeda, M. y Castro, J. (2014). Cultura y liderazgo escolar: factores claves para el desarrollo de la inclusión educativa. Actualidades Investigativas en Educación, 14(3), 1-23.

Padrós, M., Duque, E. y Molina, S. (2011) Aportaciones de la investigación europea includ-ed para la reducción del abandono escolar prematuro. Revista de la Asociación de Inspectores de Educación de España, 14, 11-17.

Pérez-Cobacho, J. y Prieto, M.D. (2003). Más allá de la integración: hacia la escuela inclusiva. Murcia: Universidad de Murcia.

Pino, J. y Castaño, M. (2019). Pertinencia y actualidad de la pedagogía amigoniana. Revista Colombiana de Educación, 77, 1-22. DOI: 10.17227/rce.num77-4925.

Rodríguez, H. (2015). Marco de referencia internacional y modelos emergentes en educación inclusiva: análisis sobre el enfoque inclusivo en educación. En A. Escarbajal (ed.), Comunidades Interculturales y democráticas (pp. 39-52). Madrid: Narcea.

Sáez, J.M. (2017). Investigación educativa. Fundamentos teóricos, procesos y elementos prácticos. Madrid: Universidad Nacional de Educación a Distancia (UNED).

Santos Rego, M.A. (2004). La investigación educativa en Galicia (19892001). Santiago de Compostela: Xunta de Galicia.

Sarrado, J.J., Cléries, X., Ferrer, M. y Kronfly, E. (2004). Evidencia científica en medicina: ¿única alternativa? Gac. Sanit., 18(3), 235-244.

Toro, I.D y Parra, R.D. (2006). Método y conocimiento metodología de la investigación. Medellín: Fondo Editorial Universidad EART.

Unesco (2005). Guidelines for inclusion: Ensuring Access to Education for All. París. Recuperado de http://unesdoc.unesco.org/images/0014/001402/140224e.pdf

Unesco (2006). Convención sobre los derechos de las personas con discapacidad. Recuperado de http://www.un.org/esa/socdev/enable/ documents/tccconvs.pdf 